\title{
La objeción de Aristóteles a la teoría platónica de la reminiscencia
}

Aristotle's Objection to the Platonic Theory of Reminiscence

Alejandro Farieta
Universidad Agustiniana (Colombia)

Recibido: 2015-11-10

Enviado a pares: 2015-11-10

Aceptado por pares: 2015-12-10

Aprobado: 2015-12-10

Pensamiento y Cultura | ISSN: 0123-0999 | eISSN: 2027-5331

pensam.cult | Vol. 18-2 | Diciembre de 2015 | pp. 6-28

DOI: 10.5294/pecu.2015.18.2.1

Para citar este artículo/To reference this article

Farieta, Alejandro. 2015. "La objeción de Aristóteles a la teoría platónica de la reminiscencia”, Pensamiento y Cultura 18 (2): 6-28. DOI: 10.5294/pecu.2015.18.2.1 


\section{La objeción de Aristóteles a la teoría platónica de la reminiscencia}

Resumen: a continuación presentamos una interpretación de la crítica de Aristóteles a la solución que ofrece Platón a la Paradoja del Menón. Según Aristóteles, cuando Platón dice que se da la reminiscencia (anámnēsis), realmente lo que se da es inducción (epagōgê). Nuestra interpretación se centra en dos aspectos: (1) la crítica semántica, pues el uso que hace Platón del término anámnēsis no es habitual; y (2) la teoría no es capaz de dar una adecuada explicación del efectivo descubrimiento.

Palabras clave: Platón; Aristóteles; Paradoja del Menón; reminiscencia; inducción.

\section{Aristotle's Objection to the Platonic Theory of Reminiscence}

Abstract: This paper provides an interpretation of Aristotle's criticism to the solution to Meno's Paradox suggested by Plato. According to Aristotle, when Plato says that reminiscence (anámnèsis) is achieved, what is actually achieved is induction (epagogêे). Our interpretation is based on two aspects: (1) semantic criticism, since Plato's use of the term anámnēsis is unusual; and (2) the theory is not able to give an adequate explanation of the effective discovery.

Keywords: Plato; Aristotle; Meno's Paradox; reminiscence; induction. 


\section{Introducción}

Uno de los primeros problemas epistemológicos a los que se enfrenta Platón en sus diálogos es la Paradoja del Menón (Men.80d5-e5). La paradoja, o el problema, consiste, para presentarlo brevemente, en un dilema constructivo que puede ser presentado de la siguiente manera:

1. Si alguien sabe algo, no lo investiga — pues ya lo sabe-.

2. Si no lo sabe, tampoco lo investiga — puesto que no sabe qué ha de investigar-.

3. No investiga.

La solución que ofrece Platón para resolver este problema está sustentada por la Teoría de la reminiscencia, según la cual el alma, antes de unirse con el cuerpo, ya conoce todo y, de este modo, en cualquier proceso de investigación y de adquisición de nuevo conocimiento - i.e., de «aprendizaje»— el alma no «aprende», sino que «recuerda». ${ }^{1}$ Esta tesis platónica es repetida en textos posteriores al Menón (Phd. 72e-74d), y es asociada generalmente con otras tesis platónicas como la de la inmortalidad del alma o incluso la teoría de las ideas (Phd. 72e-77e). Generalmente se considera que la teoría de la reminiscencia es una parte central de, o un primer avance de Platón en dirección a, la teoría de las ideas, y que ambas se encuentran estrechamente relacionadas. ${ }^{2}$

Son bien conocidas las críticas que hace Aristóteles a la Teoría de las ideas en general; a las cuales dedicó el tratado Peri Ideon-que se conserva en la actualidad solamente de manera fragmentaria (cf. Fine, 1993) - , aunque también se encuentran en otras partes del corpus (Metaph. I, 9; NE I, 6; EE I, 5). Pese a esto, no es tan conocida la crítica y el análisis que hace Aristóteles de la Teoría de la Reminiscencia como parte de la Teoría de las ideas, aunque puede considerarse como un punto importante en el que se separa de su maestro.

1 Presentamos con más detalle una interpretación de la Paradoja del Menón en Farieta (2013).

2 Así lo afirman, entre otros, Crombie ([1964] 1979, 142-148), Annas (2003, 8-10) y Correa (2003). 
En este ensayo presentaremos una interpretación de la crítica hecha por Aristóteles a la Teoría de la reminiscencia. Dicha crítica se encuentra explícita en Analíticos Primeros II, 21, por lo que la primera parte de este ensayo estará dedicada mostrar por qué esta crítica se encuentra en este tratado - dedicado la lógica- aun cuando la teoría es de un carácter eminentemente epistemológico. Así, intentaremos mostrar que hay ciertos problemas de la teoría que no son, según Aristóteles, propiamente epistemológicos, sino más bien de carácter lógico.

En la segunda y tercera parte del ensayo mostraremos que también hay ciertos problemas de tipo epistemológico en la teoría, pero que Aristóteles no hace completamente explícitos. Para ello recurriremos a otras partes del corpus, explícitamente al breve tratado De Memoria et Reminiscentia en el que Aristóteles presenta su propia versión de lo que se considera "recordar". Como veremos, esta versión aristotélica evidencia otros problemas adicionales en la teoría platónica, ya no tanto en el terreno de la lógica, sino más bien en el campo de la explicación; en particular, en la capacidad de la teoría platónica de explicar cómo es posible adquirir conocimiento, problema que fue justamente el que hizo que Platón propusiera esta teoría.

\section{Lógica y epistemología}

El capítulo 21 del libro II de los Analíticos Primeros -donde se encuentra la crítica a la teoría de la reminiscencia de Platón- Aristóteles inicia analizando los errores que pueden tener lugar al efectuarse una deducción (syllogismós) ${ }^{3}$. Dado que en los capítulos precedentes de esta obra Aristóteles ha desarrollado su teoría de la deducción, la pregunta es entonces, ¿qué tiene que ver un problema como la Paradoja del Menón con el desarrollo puramente lógico que hace Aristóteles en este libro (por lo menos hasta este momento)? La respuesta a esta pregunta, como veremos más adelante, es que la Teoría de la reminiscencia da lugar a

3 Es preciso aclarar de entrada la ambigüedad que existe en la lógica aristotélica con respecto a este término, pues bien puede tratarse de la entidad puramente lingüística, o del proceso mental y psicológico. Con respecto a la escogencia del término "deducción" para traducir

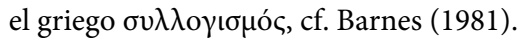


problemas lógicos al asumir que se sabe y no se sabe al mismo tiempo, pues no parece hacer una clara distinción entre dos formas de saber.

Por ahora detengámonos en el contenido de este capítulo, el cual parece girar en torno a la opinión según la cual a veces se puede saber y a la vez ignorar la misma cosa, sin que esto implique, al parecer, que haya una contradicción de opiniones. El primer error que se analiza (APr., 66b27) es el caso de alguien que sabe que todo A se da en $B$, y que todo $\mathrm{B}$ se da en $\mathrm{D}$, sin embargo, ignora que $\mathrm{A}$ también se da en $\mathrm{C}$, ya que sabe que todo $\mathrm{C}$ se da en $\mathrm{D}$, por lo tanto no cree que $\mathrm{C}$ se de en $\mathrm{D}$. Lo que tendríamos, en conclusión, es una paradoja del siguiente tipo:

A se da en todo B

$B$ se da en todo D

A se da en todo D (por $\{1\}$ y $\{2\}$ )

$S$ sabe que $\{1\}$

$S$ sabe que $\{2\}$

$S$ sabe que $\{3\}$ (por $\{4\}$ y $\{5\}$ )

A se da en todo $C$

C se da en todo D

A se da en todo D (por $\{7\}$ y $\{8\}$ )

$S$ no sabe que $\{7\}$

$S$ no sabe que $\{8\}$

$S$ no sabe que $\{9\}$

- $S$ sabe que «A se da en todo $\mathrm{D} »(\{\mathrm{~A} 6\})$ y a su vez, no sabe que «A se da en todo $\mathrm{D} »(\{\mathrm{~A} 9\})$

Obviamente el error en esta supuesta paradoja depende del desconocimiento por parte de S de una de las cadenas en la inferencia. La paradoja se deshace fácilmente al señalar que el conocimiento que $S$ tiene de $\mathrm{D}$ proviene de la relación entre $\mathrm{D}, \mathrm{A}$ y $\mathrm{B}$, mientras que la ignorancia proviene de la relación entre $\mathrm{A}, \mathrm{C}$ y $\mathrm{D}$.

El segundo error es muy similar al anterior, lo que cambia es que ahora se ignora un eslabón en la cadena de razonamientos A-B-C-D. Si se supiera que $A$ se da en todo B y que B se da en todo C, pero se creyera que $A$ no se da en ningún $C$, parecería que igualmente se cree que $A$ se da en todo $\mathrm{C}$ y que A no se da en ningún C. Así pues, dice Aristóteles: 
"Se sabe, en efecto, que de alguna manera A se da en C a través de B, así como se da el saber particular a través del universal" (APr., 66b31-33). ${ }^{4}$ El error que se presenta aquí es un error casi por descuido: fue simplemente un error al hacer la inferencia, o mejor, al no realizar la inferencia por creer que A no se da en ningún C; $\mathrm{o}$, tal vez, al tener unas razones independientes a esta cadena de inferencias para creer que A no se da en ningún $\mathrm{C}$.

Un elemento importante para subrayar es la afirmación "de alguna manera se sabe”. ¿Qué manera es esta? Según lo dicho por Aristóteles, es de la misma manera en que se sabe lo particular a través de lo universal, pero hasta este punto el texto no es preciso en hacer la distinción entre lo universal y lo particular.

El siguiente ejemplo de error aparece en (APr., 67a9-12). Es el error respecto a los particulares, lo cual nos hace ver cierto parecido con el ejemplo que se había mencionado previamente. El ejemplo es el siguiente:

Si A se da en todo B, y B en todo C, A se dará en todo C; así pues, si alguien sabe que $\mathrm{A}$ se da en todo $\mathrm{B}, \mathrm{y}$ que $\mathrm{B}$ se da en todo $\mathrm{C}$, sabrá que $A$ se da en todo $C$; pero es posible que no sepa que exista $C$; e.g., sea $\mathrm{A}$ : dos ángulos rectos <como suma de sus ángulos internos>, B: triángulo, y $C$ : triángulo sensible. Es posible que suceda que alguien no sepa que existe $\mathrm{C}$, y que sepa que todo triángulo tiene dos ángulos rectos <como suma de sus ángulos internos $>$, y por lo tanto sabría e ignoraría a la vez esto mismo (APr., 67a9-16). ${ }^{5}$

Analicemos esto con un poco de detenimiento:

\{B1\} Todo triángulo tiene dos ángulos rectos < como suma de sus ángulos internos>

\{B2\} Este es un triángulo

4 A menos que se indique lo contrario, todas las traducciones son nuestras.

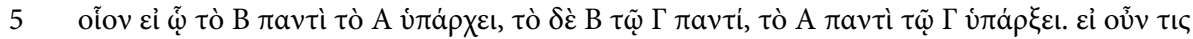

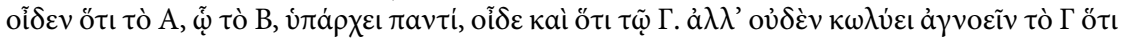

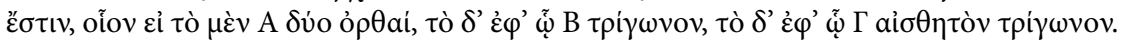

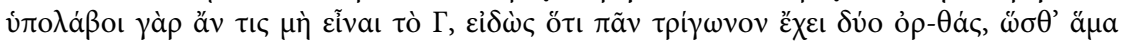

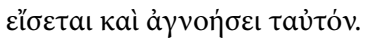


\{B3\} Este tiene dos ángulos rectos < como suma de sus ángulos internos>.

Hasta aquí el razonamiento no tiene mayor problema; el problema aparece cuando tenemos que introducir un sujeto cognoscente. Con esta inclusión aparece una paradoja similar a la que mencionábamos antes, que continuaría así:

$\{$ B4 $\}$ sabe que $\{\mathrm{B} 1\}$

$\{\mathrm{B} 5\} \quad S$ no sabe que $\{\mathrm{B} 2\}-$ En sentido de no saber que existe este triángulo; i.e., de no haberlo visto nunca antes-

$\{$ B6 $\} S$ no sabe que $\{$ B3 $\}$

$\{\mathrm{B} 7\}$ sabe que "si $\{\mathrm{B} 1\}$ y $\{\mathrm{B} 2\}$, entonces $\{\mathrm{B} 3\}$ "

$\{\mathrm{B} 8\} S$ sabe que $\{\mathrm{B} 3\}$ (por $\{\mathrm{B} 4\}$ y $\{\mathrm{B} 7\}$ )

- $S$ sabe que $\{\mathrm{B} 3\}-$ por $\{\mathrm{B} 8\}-y$ no sabe que $\{\mathrm{B} 3\}-$ por $\{\mathrm{B} 6\}-$

Observando detenidamente este esquema, podemos detectar una falacia al concluir $\{B 8\}$. Es diferente saber que «si $p$ entonces $q »$ a conocer cada una de las partes del enunciado, a saber, $p$ y $q$. El conocimiento que $S$ tiene en $\{\mathrm{B} 8\}$ es un conocimiento general e hipotético, pero no es un conocimiento particular de facto, como sí lo es saber $\{\mathrm{B} 2\}--$ como se indica en $\{\mathrm{B} 5\}$ -

Aristóteles resuelve esta paradoja de la siguiente manera:

Por otra parte, no es unívoco <el hecho de> saber que todo triángulo tiene dos ángulos rectos <como suma de sus ángulos internos $>$, sino que de uno lado se sabe de manera universal, y de otro, de manera particular (APr., 67a16-19). ${ }^{6}$

Aquí aparece la distinción fundamental para deshacer este tipo de paradojas: se conoce, por una parte, de manera universal — como $S$ sabe que $\{B 3\}$ en $\{B 8\}-$, pero por otra parte, se sabe $-o$ se ignora- de manera particular — como $S$ no sabe que $\{\mathrm{B} 3\}$ en $\{\mathrm{B} 6\}-$. Esta paradoja es llamada por Gifford la "Paradoja del conocimiento de universales"

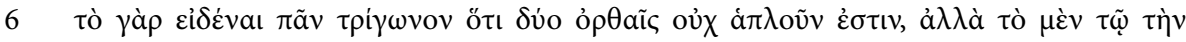

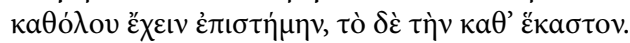


(Gifford 1999, 3-5) la cual depende de un principio que aparece en APr. 67a9-12, al que este mismo autor llama el Principio de instanciación universal en contextos cognitivos (Principle of universal instantiation in knowledge-contexts, UIK Principle). Este principio es planteado por Gifford de la siguiente manera:

$S$ sabe que todo $\mathrm{B}$ es $\mathrm{A}$

$c$ es un $\mathrm{B}$

- $S$ sabe que $c$ es A (Gifford, 1999, 3).

Este principio lo podemos ver en la esquematización de la paradoja que planteamos anteriormente en $\{\mathrm{B} 7-\mathrm{B} 8\}$, y podemos prever que es el punto al que Aristóteles apunta al desarmar la paradoja. Gifford señala que este principio es ambiguo por varias razones. La primera de ellas es que la primera premisa " $S$ sabe que todo B es $A$ " se puede interpretar de varias maneras, tal como Aristóteles lo señala al afirmar que "Saber que todo B es A no es unívoco" (APr., 67a16, supra).

De acuerdo con Gifford, la solución aristotélica de la Paradoja del conocimiento de universales depende principalmente de cuatro tesis:

(T1) Un reporte de conocimiento de la forma " $S$ sabe que todo B es A" es ambiguo entre la adscripción de universales y el conocimiento enumerativo.

(T2) El conocimiento explícito de una verdad universal no implica conocimiento explícito de toda afirmación singular que caiga sobre tal verdad; esto es, el conocimiento universal no implica conocimiento enumerativo (esto representa el rechazo de Aristóteles del Principio de instanciación universal en contextos cognitivos, cuando su premisa mayor es leída como adscribiendo conocimiento universal).

(T3) Los seres humanos pueden tener conocimiento explícito de afirmaciones universales; esto es, ellos pueden poseer conocimiento universal.

(T4) El Principio de instanciación universal en contextos cognitivos implica conocimiento potencial de cada afirmación singular que cae bajo la verdad universal conocida (Gifford 1999, 8). 
En nuestraopinión,(T2) - dela que depende principalmentela desarticulación de la paradoja del conocimiento de universales- merece un adendum para evitar ciertos equívocos: el conocimiento de universales no implica, sin más, el conocimiento de particulares; pero de alguna manera sí debe servir para adquirir conocimiento sobre estos. La razón de esto es muy sencilla: si los dos tipos de conocimiento fueran totalmente separados, y no fuera posible establecer relación entre los dos, entonces el conocimiento universal no sería útil al momento de hacer inferencias sobre los particulares. En otras palabras, los universales, tomados de esta manera, no tendrían ninguna relación epistémica con los particulares, ya que al conocer un universal no sabríamos nada sobre los particulares que serían las instancias de aquellos. La tesis (T2) debe aceptar cierto tipo de relación epistémica entre universales y particulares; la relación entre estos dos tipos de conocimiento debe dar cuenta de que el conocimiento universal depende del particular en cuanto no se puede dar el primer tipo de conocimiento si no hay un conocimiento particular al cual aplicarle conocimiento universal; por lo tanto, el conocimiento universal implica cierto conocimiento particular siempre y cuando se tenga ya de antemano algún tipo de conocimiento particular. De esta manera, el conocimiento universal vale como conocimiento hipotético sobre los particulares, mientras estos últimos no estén presentes.

Aclaremos esto basándonos en el ejemplo: cuando decimos que todo triángulo tiene dos ángulos rectos como suma de sus ángulos internos, este conocimiento sólo podrá tener carácter hipotético mientras no se tenga un triángulo sensible al cual aplicarle el conocimiento universal, para que, luego de haberlo percibido, sí sea posible concluir que tal triángulo sensible tiene dos ángulos rectos como suma de sus ángulos internos. La tesis de Aristóteles sería, hechos los arreglos, la siguiente:

(T2A): El conocimiento de universales implica el conocimiento de particulares si y sólo si existen particulares a los cuales se pueda

7 Cabe aclarar que cuando nos referimos al conocimiento de universales como conocimiento hipotético no queremos negar que tal conocimiento tenga carácter universal y necesario, como Aristóteles lo va a señalar luego (APo., A, 4); simplemente queremos afirmar que este tipo de conocimiento no tiene validez epistémica mientras no hayan particulares sensibles a los cuales se les pueda suscribir tales características universales. 
aplicar dicho conocimiento universal; mientras tanto, sólo servirá como conocimiento hipotético acerca de estos últimos.

La principal característica que asegura esta tesis es que con el conocimiento universal no es posible, sin más, admitir la existencia de particulares; como observa Aristóteles en varias oportunidades (Int., 16b18-26), una afirmación del tipo "A se da en todo B" no implica sin más que exista $\mathrm{B}-$ ni siquiera que exista $\mathrm{A}-$. La distinción fundamental entre el conocimiento universal y el particular es que el segundo necesita, como en el caso del triángulo, de la experiencia sensible del objeto particular, i.e., de aisthêsis. Sin esto, todo conocimiento universal es un conocimiento acerca de nada - en principio, tal conocimiento no debería ni siquiera tener lugar, como lo muestra el Estagirita posteriormente en esta misma obra ( $A P r$., $\mathrm{B}$, 23), y también en los Analíticos Posteriores (APo., A, 18).

\section{Epagōgê}

Luego de resolver la paradoja del conocimiento de universales, Aristóteles presenta su argumento contra la tesis platónica de la Teoría de la reminiscencia señalando lo siguiente:

Igualmente < sucede > con el argumento del Menón, según el cual el aprendizaje es reminiscencia. Porque de ninguna manera sucede que lo particular se conozca previamente, sino que se adquiere conocimiento de lo particular junto con la inducción (epagōgê), como si se reconociera. Pues algunas cosas las sabemos inmediatamente, como que <este triángulo> tiene dos ángulos rectos $<$ como suma de sus ángulos internos $>$, si sabemos que es un triángulo, e igualmente con las demás (APr., 67a21-26). ${ }^{8}$

Aquí Aristóteles indica cuál es ese proceso que señalamos antes, a saber, el proceso de identificación entre el conocimiento universal y los

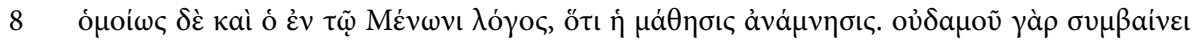

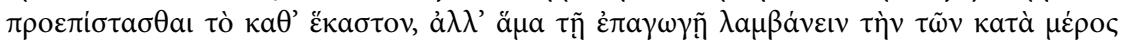

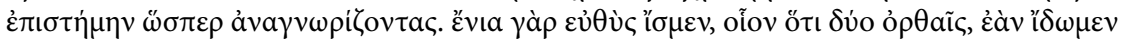

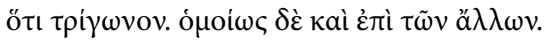


particulares que entran en su dominio. A ese proceso lo llama inducción (epagōgêe). ${ }^{9}$ Adicionalmente, lo que tenemos en el ejemplo anterior parece, a primera vista, ser un caso de deducción con una premisa mayor universal $\{\mathrm{B} 1\}$ y una premisa menor particular $\{\mathrm{B} 2\}$, que tiene como conclusión un enunciado particular $\{B 3\}$. En varias oportunidades Aristóteles opone inducción y deducción (APo., I, 1, 71a4-5), sin embargo, como parece seguirse del texto, estas no se oponen radicalmente, pues ambas funcionan a la vez: la deducción nos permite inferir la conclusión, y esto parece no tener mayor problema, mientras que la inducción sería esa identificación de la premisa $\{\mathrm{B} 2\}$. La identificación del objeto particular con la clase a la que pertenece es lo que Aristóteles está llamando aquí epagōgê; la inducción, por lo tanto, no es en Aristóteles - como lo fue en la Modernidad - un proceso que nos conduce de un hecho particular a una ley universal, sino que simplemente nos permite establecer la relación entre universales y particulares.

Recordemos brevemente cuál era el problema que Platón pretendía resolver con la anámnēsis, y así podremos entender por qué Aristóteles reemplaza ésta con la epagōgê. ${ }^{10}$ En primer lugar leamos la versión abreviada de la tesis platónica:

9 Como lo han señalado varios autores, entre ellos Hintikka (1980), Smith (1989), Caujolle-Zaslawsky (1990), traducir epagōgê por "inducción” sin dar una explicación puede conducir a un error enorme, dada la connotación que tuvo este término principalmente en los filósofos modernos. En este momento no vamos a dar tal explicación, ya que consideramos que es mejor que ésta vaya apareciendo a medida que analicemos los diversos fragmentos en los cuales se utiliza; sin embargo, mantenemos el término 'inducción' y no 'comprobación' que utiliza Candel en su traducción a los Tratados de Lógica $(1982,1988)$, expresión poco aceptada y muy difícil de comprender, pues no es claro qué es lo que se comprueba. Como defenderemos más adelante, la principal función de la epagōgê es la de descubrir. Por el momento, y siguiendo a Hintikka, es posible aceptar 'inducción' si entendemos ésta básicamente en su sentido etimológico: "ser inducido hacia", o "ser llevado hacia".

10 Gifford apunta que, al Aristóteles lidiar con la tesis de la reminiscencia platónica, lo que afirma es "no, en lugar de la reminiscencia lo que tiene lugar es la inducción" $(1999,15)$. Fine lee este pasaje de manera sustancialmente distinta, pues afirma que no se trata explícitamente de una crítica a la teoría platónica de la reminiscencia (2010, 213-215), sino de un caso de reconocimiento a Platón por haber evidenciado un caso de «quasi-reminiscencia» como el de esclavo, pero no de reminiscencia en sentido estricto (cf. Vlastos, 1965). Bronstein (2010, 138-139) también presenta una lectura más caritativa y menos crítica con Platón de este pasaje. 
puesto que la naturaleza [phýsis] es toda congénita de sí misma, y puesto que el alma [psýkhe] ha aprendido todo, nada impide que aquel que recuerde una sola cosa - eso que los hombres llaman aprendizaje-, descubra él mismo todo lo demás, si es valiente y perseverante al buscar. En efecto, buscar y aprender son tan solo reminiscencia (Platón, Men. 81c9-d5). ${ }^{11}$

La tesis consistía en que no había aprendizaje sino reminiscencia, y esto se sostenía porque la psýkhe podía tener conocimiento de la phýsis, y tal conocimiento estaba preexistente en aquélla. Aristóteles, con este pasaje en mente, insiste en que sí es posible tener conocimiento de universales, pero subraya que al utilizar la phýsis para señalar dicha posibilidad se está incurriendo en un craso error al creer que se puede tener conocimiento sensible de ésta previamente a cualquier sensación; de no aceptar esto, entonces se tendría que aceptar que el conocimiento sensible no tiene ninguna validez epistémica, lo cual parece ser la opción que toma Platón, por lo menos en el Menón. ${ }^{12}$ Ya que la tesis de la anámnēsis toma por sentado que hay, de hecho, conocimiento previo, y Aristóteles afirma que lo particular - i.e., lo sensible - es imposible conocerlo previamente, el problema fundamental para la tesis de Platón es poder establecer un nexo entre los universales y lo sensible: ¿Es posible, para Platón, poder afirmar tal nexo entre universal y particular? Según la tesis de la reminiscencia, por lo menos en el Menón, parece que no. Podemos concluir que Aristóteles está reemplazando la teoría según la cual ya sabíamos todas las cosas - y, al aprender, simplemente recordamos- por una teoría que tiene ciertas ventajas, a saber, la epagōgê, explica cómo hacemos para que una sensación particular la podamos de alguna manera clasificar dentro de un conjunto de universales. Quizás sea relevante citar un fragmento de Metafísica en donde Aristóteles

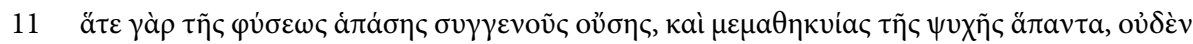

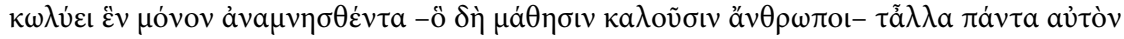

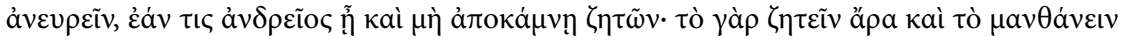

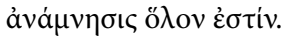

12 En textos posteriores como el Fedón o el Filebo Platón sí tiene en cuenta el hecho de que para que haya reminiscencia debe haber experiencia sensible. 
parece complementar esta crítica a la anámnēsis con una crítica directa a la Teoría de las Ideas:

[El universal] lo reveló Sócrates a través de las definiciones, aunque ciertamente no lo separó de los particulares. Y consideró correctamente esto al no separarlo. En efecto, sin lo universal no es posible captar el conocimiento científico. Esto es evidente por los resultados, pues separarlo es la causa de las dificultades que se dan con respecto a las ideas (Metaph., 1086b3-7). ${ }^{13}$

Esta parece ser, entonces, la crítica fundamental a la anámnēsis: la teoría separa irreconciliablemente a los universales con sus particulares, haciendo imposible mostrar cómo se relacionan los unos con los otros. Esto sucede debido principalmente a que no es posible establecer cómo de la percepción se llega al universal, pues si la tesis de Platón es cierta, no se necesita tener conocimiento de los particulares para alcanzar el conocimiento universal; tal conocimiento universal sería necesariamente previo. Pero ¿de dónde surgiría este conocimiento previo pero universal? Más aún, ¿cómo podríamos garantizar que estos universales, que se conocen previamente, se relacionan efectivamente con los particulares sensibles? La teoría de Platón no parece poder dar cuenta de esta conexión, simplemente la supone. Así, no es solamente que Aristóteles critique la tesis platónica porque cree que la suya sea mejor; tiene además fuertes razones para considerar que, independientemente del valor de su propia propuesta, hay algo mal con la reminiscencia.

Para finalizar, veremos con más detalle las ventajas que tiene la inducción con respecto a la reminiscencia platónica. No con el propósito de beneficiar la postura aristotélica sobre la platónica, sino para identificar, en esta presentación que hace Aristóteles, cuál es el problema o dificultad que hace que sea necesaria la aparición de la respuesta aristotélica.

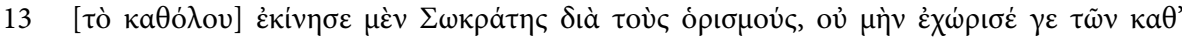

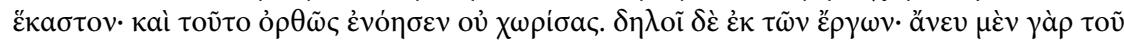

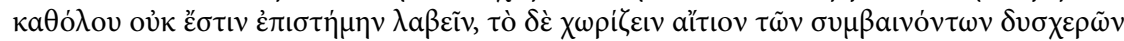

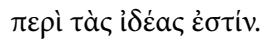




\section{Epagōgê versus anámnēsis}

Cabe señalar ahora las ventajas que tiene la epagōgê sobre la anámnēsis, las cuales hicieron que Aristóteles no aceptara esta teoría platónica. La primera de ellas tiene que ver con el uso que se le da comúnmente a la expresión anámnēsis. Veamos la definición que hace de ésta Aristóteles en su De Memoria et Reminiscentia:

La reminiscencia [anámnēsis] no es ni una readquisición ni una adquisición de memoria, pues cuando alguien aprende o experimenta por primera vez, ni recupera la memoria de nada en absoluto - ya que la memoria tampoco había actuado antes en absoluto- ni la adquiere desde el principio; y es que hay memoria cuando sobreviene el estado o la afección, de suerte que no se da cuando se produce la experiencia [...]. El recordar en sí no se produce hasta que no ha pasado un tiempo, pues se recuerda ahora lo que se vio o experimentó antes; lo que ahora se experimenta no se recuerda ahora. Además, es evidente que es posible recordar, no cuando se ha rememorado ahora, sino cuando desde el principio se ha sentido o experimentado. Pero, cuando se recupera el conocimiento científico o la sensación que antes se tenía o aquello a cuya posesión llamamos «memoria», eso es, precisamente, la reminiscencia (Mem., 451a22-b5). ${ }^{14}$

Como podemos ver, y como lo indicamos antes, solamente podemos recordar aquello de lo que tuvimos, en una ocasión pasada, una

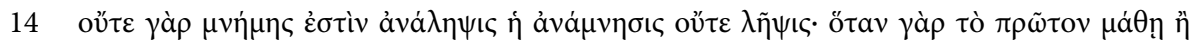

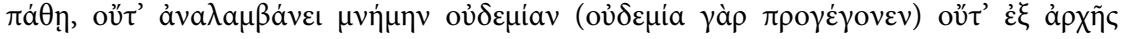

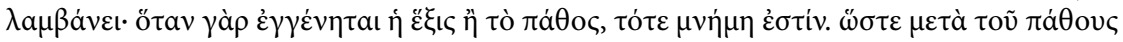

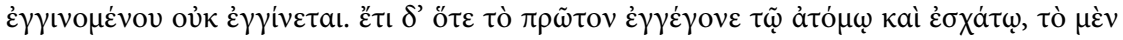

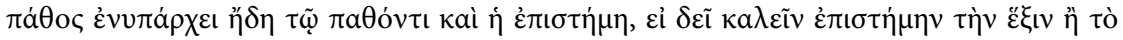

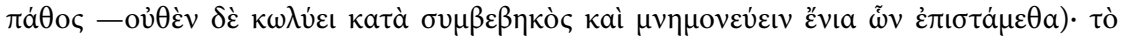

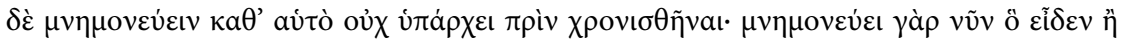

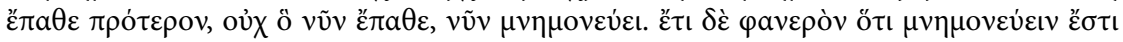

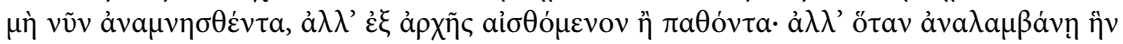

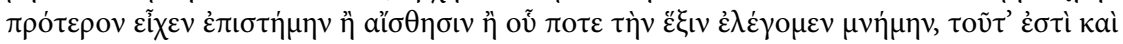

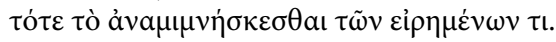


experiencia sensible y sin esto no hay reminiscencia; es por esto que para Aristóteles todo acto de rememorar exige de una experiencia sensible previa, la que luego es recordada. ${ }^{15}$ Cuando se tiene por primera vez una experiencia sensible, entonces, y volviendo a la discusión anterior, es posible no hacer anámnèsis, sino epagōgêe, pues con base en esta experiencia sensible se van conformando los conocimientos universales, que luego, con la adquisición de nuevas experiencias sensibles, se irán estableciendo más detenidamente. Es esto a lo que Aristóteles apunta en APr. II, 23 al señalar cómo funcionan los argumentos por inducción:

Asimismo, la inducción y la deducción por inducción consisten en probar, a través de uno de los extremos, que el otro $<$ se da $>$ en el medio, v.g., si B es el medio de A y C, probar que A se da en B por medio de $\mathrm{C}$; en efecto, así hacemos las inducciones. Por ejemplo, sea A larga vida, en lugar de $B$, carente de hiel, $y$, en lugar de $C$, larga vida particular, v.g. hombre, caballo, mula. Entonces A se da en todos los $\mathrm{C}$, pues todo <animal> que carece de hiel tiene larga vida; pero también B, el no tener hiel, se da en todo C. Si, pues, se invierte C respecto a $\mathrm{B}$ y el medio no tiene mayor extensión, es necesario que $\mathrm{A}$ se dé en B. (APr., 68b15-24). ${ }^{16}$

15 Esta definición de anámnēsis, si puede valer por tal, no parece una invención puramente aristotélica, sino que parece extraída del uso cotidiano de la expresión misma; esta es, más que un término técnico de la filosofía aristotélica, un legómenon, un éndoxon al cual Aristóteles trata de mantener hasta donde sea posible. Sobre esta tendencia aristotélica por defender los éndoxa, es clásico el aporte de Owen, quien muestra que en ciertos contextos, específicamente en $A P r$., el término éndoxa tiene un valor semejante al de phainómena y "los phainómena deben ser recogidos como un preludio para encontrar la teoría que los explique" (1961, 83). Si bien Aristóteles no defiende literalmente el significado de anámnēsis, sino que elabora su propia definición, mucho más técnica que la del uso corriente (Bloch, 2007, 132-135), el punto es que la interpretación aristotélica es mucho más compatible y coherente con el uso normal del término que la platónica, que más bien parece contradecir los éndoxa.

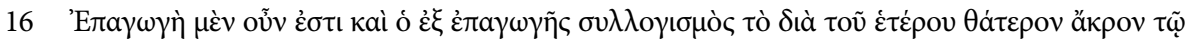

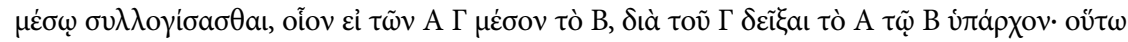

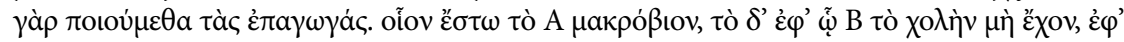

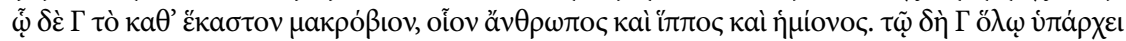

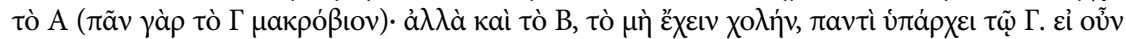

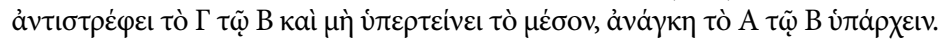


Tomemos ahora este ejemplo para analizar mejor cómo la epagōgê puede suplir, en buena medida, los requerimientos que condujeron a Platón a introducir la anámnèsis cuando trató de solucionar la Paradoja del Menón. Digamos, por ejemplo, que estamos en búsqueda de la definición de "animal de larga vida". Por experiencia empírica hemos visto que los animales que tienen larga vida son el hombre, el caballo y la mula. Podemos reconocer - a través de la epagōgê- que estos son los únicos animales que carecen de hiel, y por lo tanto, podemos explicar que, dada su carencia de hiel, estos animales tienen larga vida.

En la cita anterior Aristóteles está señalando lo que sería no una inducción sino una deducción por inducción; esto es, en cierta medida, diferente, pues, como señala Hintikka $(1980,426)$, se trata de buscar la explicación adecuada para un hecho que se toma por dado, como es en este caso el de la longevidad de ciertos animales, y con este propósito se utiliza el esquema de la deducción. ${ }^{17}$ Para Aristóteles, buscar una explicación es buscar el por qué y parece bastante insistente en que hay ciertos argumentos que pueden ser del qué y otros del porqué. El ejemplo anterior dependía de la conexión entre A (larga vida) y B (carente de hiel), y el hecho que el segundo explica el primero, de ahí que las subclases del primero sean los ejemplos que sirven para establecer la conexión necesaria entre A y B.

En Analíticos Segundos Aristóteles será más explícito con este tipo de razonamientos, en los cuales se busca explicar por medio del por que y no del que, como es el caso de dos argumentos bastante parecidos - que

17 Esto muestra que la interpretación moderna de la inducción - como razonamiento que parte de lo particular para concluir lo universal- sea desorientadora para comprender la posición aristotélica. En este caso particular, es posible llevar a cabo, al mismo tiempo, tanto una inducción como una deducción. Lo mismo sucedía en el pasaje de APr. II, 21, en donde se afirma que "se adquiere conocimiento de lo particular junto con la inducción, como si se reconociera. Pues algunas cosas las sabemos inmediatamente" (APr., 67a22-25). Esta simultaneidad de la inducción se contrapone a la mediatez de la deducción; no son, en sentido estricto, dos procesos contrarios el uno y el otro. La tesis aquí presentada se opone a la de Gifford (1999), para quien la lectura de este último pasaje debe hacerse en términos puramente induccionistas en oposición a interpretaciones clásicas como la de Ross (1949, 481-483) y McKirahan (1983), las cuales debilitan las pretensiones de Aristóteles en este texto. Por nuestra parte, consideramos que es inverosímil negarle el estatus de deducción al ejemplo presentado en ambos pasajes, y por lo tanto creemos pertinente que la opción es buscar una interpretación diferente para la epagōgê en estos pasajes. 
se encuentran en dos capítulos que tienen una importante conexión con el que estamos señalando: el ejemplo de los planetas (APo., 78a30-b4) y el ejemplo del eclipse (APo., 90a26-30)—. Veamos rápidamente estos ejemplos. En el primero de ellos el problema es saber cuál es el hecho y cuál es la explicación: es decir, saber si la explicación de por qué titilan los planetas es porque ellos están cerca o si, por el contrario, ellos están cerca porque titilan. Obviamente, la deducción del por qué es la siguiente: (B) lo que no titila (A) está cerca, (C) los planetas (B) no titilan, por lo tanto $(\mathrm{C})$ los planetas $(\mathrm{A})$ están cerca; esto es una verdadera demostración y no una simple deducción. Sin embargo, Aristóteles señala que dada la reciprocidad entre «no titilar» y «estar cerca» - que es, entre otras cosas, la misma reciprocidad que hay entre «larga vida» $\mathrm{y}$ «carente de hiel» en el ejemplo anterior-, es posible hacer una «deducción a la inversa» en la cual se concluiría que (C) los planetas (A) no titilan; no obstante esta no es una deducción que nos ofrezca una explicación - ya que no titilar es algo que podemos verificar empíricamente-, sino más bien nos señala el que, es decir, la premisa mayor, y puede resultar que el término medio no se haya escogido adecuadamente.

Este asunto de la escogencia del término medio va a tomar mayor importancia en el ejemplo del eclipse, pues allí justamente el término medio va a servir de explicación (APo., 90a11-12). Según Aristóteles, preguntar ¿cuál es la causa del eclipse? equivale a decir ¿qué es un eclipse? -que, entre otras, es análoga a la equivalencia entre las preguntas ¿por qué los animales longevos viven tantos años?; ¿qué es un animal longevo? O las preguntas ¿por qué los planetas no titilan?, ¿qué es un planeta?-. Claro está, el primer ejemplo es un tanto diferente, pues se está preguntando por un subgénero que se encuentra en el género «animal» y las diferentes especies que se señalan, i.e., «hombre», «caballo», etc. Pese a esta diferencia, el objetivo de Aristóteles con el ejemplo inductivo es que el término medio, al estar en relación tanto del término mayor como del menor, es el que sirve como explicación de por qué este tipo de especies pertenece al subgénero de los animales de larga vida.

Tenemos que separar los dos usos que se le está dando a la inducción. Por un lado, en el ejemplo del triángulo (APr., 67a9-16) se aseguraba que la inducción servía para identificar una instancia con su clase, 
cuando se identificó la premisa «este es un triángulo». Por otro lado, en los ejemplos del eclipse y del animal longevo se utiliza la inducción no para identificar una instancia con su clase, sino para buscar la explicación - y en últimas, la definición - de tal clase. ¿Es posible reconciliar estos dos usos, tan radicalmente distintos de la epagōgê? Señalemos por ahora que Aristóteles confía en que su proceso de inducción suple las desventajas que tiene la reminiscencia platónica, en particular las que tiene al tratar de buscar explicaciones y poder dar explicación de ciertos hechos, a saber: del hecho de que podamos tener conocimientos universales $y$, principalmente, del hecho de que podamos, de cierto modo, adquirir tales conocimientos; i.e., descubrirlos.

Vemos entonces que la anámnēsis platónica, además de alejarse demasiado del uso habitual del término, encuentra varios obstáculos. El principal de estos aparece cuando se tiene que aceptar, en cierta medida, que o bien se tiene conocimiento empírico previo a cualquier experiencia previa - lo cual de suyo es contradictorio- o bien que el conocimiento empírico no es un conocimiento en ninguna medida, sino que es mera opinión. Como consecuencia de esta tesis aparece una separación total entre los universales - las Formas o Ideas platónicas- y los particulares provenientes de la sensación, por lo que al asumir la tesis de la Reminiscencia, Platón queda sin poder establecer un nexo epistémico entre los dos.

Así, la crítica de Aristóteles a la teoría platónica de la reminiscencia tiene dos matices. El primero de ellos, semántico, pues Platón estaría utilizando la expresión en un sentido técnico muy poco habitual, separándose así del uso cotidiano del término. Por sí mismo este no es un problema para Platón, pues en su solución a la Paradoja del Menón estaría aclarando el significado particular de este término de difícil comprensión. Pero el segundo matiz, y tal vez el principal, tiene que ver con que en la teoría platónica se abre un abismo insalvable entre los universales y los particulares. Tal vez Platón mismo reconoció este problema e incluso lo haya intentado resolver, ${ }^{18}$ pero Aristóteles, al

18 De aquí la defensa que hace Fine de Platón frente a la crítica aristotélica (Fine, 2010, 215), según la cual Aristóteles no estaría exactamente haciendo una crítica, sino también recon- 
recurrir a la inducción para superar la teoría platónica, está señalando a su vez un problema que ésta tiene en términos explicativos, no solamente en términos puramente lógicos o metafísicos — como es el problema de la relación entre universales y particulares-.

Si bien Platón intenta vincular su teoría de la reminiscencia con la explicación causal, como parece evidenciar la parte final del Menón cuando Platón vincula la reminiscencia con un razonamiento explicativo (aitías logismós) (Men. 98a3-4), este intento queda sin fundamento cuando se acepta que para explicar se recurre a algo «aparentemente» más conocido - i.e. el universal- pero de facto menos conocido. En efecto, cuando se descubre la explicación, se halla algo que antes no era conocido; e.g., cuando en el ejemplo de los planetas, se descubre que es por estar cerca que no titilan, la explicación «estar cerca» es tan conocida - o tan cercana a la percepción- como el hecho mismo a explicar «no titilar». Para Aristóteles, la diferencia no está en cuál de los dos «datos sensibles» está más cercano al universal, como parece ser para Platón. Más bien, el punto en Aristóteles es que en toda explicación el explanans tiene que darse a partir de algo que es más conocido que el explanandum. Así, «estar cerca» es una explicación aceptable del hecho del «no titilar» de los planetas en virtud de que estamos suficientemente familiarizados con el hecho de «estar cerca». Pero lo que descubrimos es la asociación entre ambos hechos; es esto lo que es desconocido y que en el momento de la epagōgê descubrimos.

Así, la teoría platónica de la reminiscencia como explicación del descubrimiento tiene el problema de que no solamente se opone al significado estándar del término anámnēsis, sino que además estaría recurriendo, para explicar un hecho, a un explanans que no podríamos identificar; pues así como un recuerdo debe poder ser identificable en un instante y en un lugar determinado, debería suceder lo mismo

ociéndole a Platón haber descubierto el fenómeno de la epagōgê, que es, para Fine, una quasi-reminiscencia. Hay que sumar a esto el hecho de que en otros textos platónicos (Fedón, Filebo e inlcuso Timeo) se pueden encontrar pasajes en los que Platón intenta con mucho más detalle vincular el conocimiento de los universales con el de los particulares. Pero valga para nuestra presentación para señalar que, tal como lo presenta Aristóteles, el problema semántico parece conducir a Platón al problema epistemológico. 
con el explanans. Pero dado que lo que tenemos es un descubrimiento, es imposible que dicha identificación pueda ocurrir. Si Platón intenta genuinamente solucionar la Paradoja del Menón, debe recurrir a algo que genuinamente se desconocía antes, pero no a algo que, en cierto sentido, ya antes se conocía - aunque no seamos capaces de identificar cuándo o cómo conocíamos-.

\section{Conclusión}

Aristóteles reconoce en la tesis platónica un avance con respecto al reconocimiento del problema de la relación entre los particulares y los universales, pero al presentar una nueva respuesta está aceptando también que no está de acuerdo con la teoría de la reminiscencia. Si bien la lectura de Aristóteles es, en parte caritativa, ${ }^{19}$ su inconformidad con la teoría supone - a su vez- que ésta debe tener alguna crítica aunque no esté completamente evidente y directa en el pasaje de $A \operatorname{Pr}$ II, 21. Así, el problema lógico con el que Aristóteles asocia la teoría de la reminiscencia en este capítulo no presenta enfáticamente las razones para rechazar la anámnēsis en favor de la epagōgê; sin embargo, sí supone que la superación de la primera implica una crítica que se concentra, como hemos intentado mostrar, en un primer aspecto semántico y un segundo aspecto epistemológico de corte explicativo.

El problema lógico al que puede dar lugar la anámnēsis es significativo, pero no es suficiente para rechazar la teoría; por lo que, para dar cuenta de por qué Aristóteles rechaza esta teoría hemos intentado mostrar que hay razones mucho más de fondo, principalmente de tipo epistemológico. Estas razones tendrían que ver con el vínculo explicativo que hay entre universales y particulares. Para Platón este vínculo simplemente se «presupone», pero la mera anámnēsis es incapaz de dar cuenta de cómo están relacionados unos y otros. Aristóteles concluye que, para defender o sustentar tal vínculo debe haber una conexión mucho más sólida que la simple presuposición. De aquí que Aristóteles por lo general invierta el método explica-

19 Como lo suponen principalmente Ross (1949, 471), Fine (2010, 214-215) y Bronstein (2010, 138-139). 
tivo, afirmando en repetidas ocasiones que hay que comenzar de "lo más cognoscible para nosotros", culminando en "lo más cognoscible en sî" (APo. 71b33-72a4; EN, 1195b2-4), y al llegar a "lo más cognoscible en sí" se habrá alcanzado la explicación de aquello que percibimos.

Con la epagōgê Aristóteles quiere dar cuenta de ese proceso que conduce a la «causa» —o «explicación (aitía)» y no simplemente presuponerlo como si se diera a priori, tal como parece presuponer la teoría platónica. De esta manera, si lo que se pretende explicar es la posibilidad del descubrimiento de nuevo conocimiento, no se puede apelar sin más a un conocimiento preexistente de ninguna naturaleza; pues esto sería una petición de principio. Así que, si el proceso de la anámnēsis supone cualquier forma de conocimiento preexistente, no hay forma de eliminar la petición de principio; por lo que resultaría preferible llamar a la operación mental que se da en el descubrimiento de otra manera: epagōgê. Esta sería, por lo tanto, el proceso fundamental para el descubrimiento de nuevos conocimientos, pero estos nuevos conocimientos tendrían fundamentalmente un carácter explicativo. De aquí que Aristóteles afirme que la epagōgê es el proceso como se descubren principios, y es justamente la tarea de descubrir principios la que posibilita cualquier modo de explicación según Aristóteles. Según el filósofo, descubrir es descubrir causas, explicaciones y principios. Cualquier otro conocimiento no se descubre, simplemente se percibe.

\section{Bibliografía}

Annas, Julia. Plato. A Very Short Intorduction. Oxford: Oxford University Press, 2003.

Aristóteles. "Analytica Posteriora" [APo]. En Aristotle's Prior and Posterior Analytics, 71-100.

Aristóteles. "Analytica Priora" [APr]. En Aristotle's Prior and Posterior Analytics, 24-70.

Aristóteles. Aristotle on Memory and Recollection. Text, translation, interpretation and reception in western scholasticism [Mem.]. Ed. \& Trad. D. Bloch. Leiden \& Boston: Brill, 2007.

Aristóteles. Aristotle's Prior and Posterior Analytics. Ed., introd. \& notas

W. D. Ross. Oxford: Oxford University Press, 1949. 
Aristóteles. "De Interpretatione" [Int.]. En Categoriae et Liber de Interpretatione, editado por L. Minio-Paluello, 16-24. Oxford: Oxford University Press, 1949.

Aristóteles. Ethica Nicomachea [EN]. Ed. I. Bywater. Oxford: Oxford University Press, 1894.

Aristóteles. Ethica Eudemia [EE]. Ed. R. R. Mingay, \& J. M. Walzer. Oxford: Oxford University Press, 1991.

Aristóteles. Metaphysica [Metaph.]. Editado por Werner Jaeger. Oxford: Oxford University Press, 1957.

Aristóteles. Physica [Phys.] Editado por W. D. Ross. Oxford: Oxford University Press, 1966.

Barnes, Jonathan. "Proof and the syllogism". Cap. 1 de Aristotle on science. The "Posterior Analytics". Proceedings of the Eight Symposium Aristotelicum Held in Padua From September 7 to 15, 1978, editado por Enrico Berti, 17-59. Padua: Antenore, 1981.

Bernabé, Alberto, trad. "Acerca de la memoria y la reminiscencia”. En Aristóteles. Acerca de la generación y la corrupción - Tratados breves de historia natural., 233-255. Madrid: Gredos, 1987.

Bronstein, David "Meno's Paradox in Posterior Analytics I, 1". Oxford Studies in Ancient Philosophy 38 (2010): 115-141.

Calvo M., Tomás, trad. Aristóteles. Metafisica. Madrid: Gredos, 1994.

Candel S., Miguel, trad. Aristóteles. Tratados de lógica (Órganon). 2 vols. Madrid: Gredos, 1982, 1988.

Caujolle-Zaslawski, Françoise. "Étude preparatoire à une interprétation du sens aristotéliciénne d' Epagogé”. En Biologie, Logique et Métaphysique chez Aristote, editado por D. Devereux y P. Pellegrin, 365-387. Paris: CNRS, 1990.

Correa, Alfonso. "Una introducción a la teoría de las ideas de Platón". En: Lecciones de filosofía. Ed. L. E. Hoyos. Bogotá: Universidad Externado de Colombia, 2003.

Crombie, Ian. An examination of Plato's Doctrines. 2 vols. London: Rouletdge \& Kegan Paul, 1964. (Trad. Análisis de las doctrinas de Platón. A. Torán y J. C. Armero. Madrid: Alianza. 1979). 
Farieta, Alejandro. "Conocimiento, descubrimiento y reminiscencia en el Menón de Platón”. Universitas Philosophica 30.60 (2013): 205-234.

Fine, Gail. The Possibility of Inquiry: Meno's Paradox from Socrates to Sextus. Oxford: Oxford University Press, 2010.

Gifford, Mark. "Aristotle on Platonic Recollection and the Paradox of Knowing Universals: Prior Analytics B.21 67a8-30". Phronesis 44, ${ }^{\circ}$ 1 (1999): 1-29.

Hintikka, Jakko. "Aristotelian induction". Revue internationale de philosophie 34.133-134 (1980): 163-223.

McKirahan, R. "Aristotelian Epagoge in Prior Analytics 2.21 and Posterior Analytics 1.1". Journal of the History of Philosophy 21.1 (1983): $1-13$.

Owen, G.E.L. “Títhenai ta phainómena”. En Aristote et les problèmes de méthode, editado por S. Mansion, 83-103. Louvain: Publications Universitaires de Louvain, 1961.

Platón. Menón [Men.]. Vol. 3, de Platonis Opera, 70-100. Ed. J. Burnet. Oxford: Oxford University Press, 1902.

Platón. Fedón [Phd.]. Vol. 1, de Platonis Opera, 57-118. Ed. J. Burnet. Oxford: Oxford University Press, 1900.

Smith, Robin, ed. \& trad. Aristotle Prior Analytics. Cambridge: Hackett, 1989.

Vlastos, Gregory. “Anamnesis in the Meno". Dialogue 4.2 (1965): 143-67. 